\title{
Sensitivity Enhancement during Electrochemical Specular
}

\section{Reflectance Measurements}

\author{
ELISABET AHLBERG, DAVID P. PARKER and VERNON D. PARKER *
}

Organic Chemistry Laboratories, Norwegian Institute of Technology, University of Trondheim, N-7034 Trondheim-NTH, Norway

The effect of the magnitude of the angle of incidence of light directed toward the surface of a platinum electrode upon the absorbancetime curves measured during the chronoabsorptometric reduction of perylene in $N, N$. dimethylformamide was determined. Relative to that at normal incidence, the absorbance due to the anion radical at $582 \mathrm{~nm}$ after $2 \mathrm{~ms}$ for angles of 45,15 and $5^{\circ}$ was found to be equal to $2.8,6.9$ and 22 , respectively, in agreement with the ratios of calculated path lengths. At an angle of incidence of about $1^{\circ}$ the absorbance relative to that at normal incidence was observed to be approximately 100 and it was possible to observe absorbance-time curves for the generation of perylene anion radical in single $100 \mu \mathrm{s}$ pulses. The latter corresponds to an improvement in time resolution of the order of $10^{4}$ compared to transmission experiments at optically transparent electrodes. The construction of simplified spectroelectrochemical cells is described.

The absorbance due to an intermediate generated at an optically transparent electrode during a transmission experiment is given by eqn. (1). The subscripts refer to reactant (A) and product (B)

$A_{\mathrm{B}}=(2 / \pi) C^{\circ} \varepsilon_{\mathrm{B}} D_{\mathrm{A}}^{\frac{1}{\frac{1}{2}} t^{\frac{1}{2}}}$

For an intermediate with molar extinction coefficient $\varepsilon_{B}$ generated from substrate with diffusion coefficient $D_{\mathrm{A}}$ at an initial concentration $C^{\circ}$, the absorbance $A_{\mathrm{B}}$ increases linearly with the square root of time.1,2 Enhanced absorbance is observed during internal reflectance experiments at optically transparent electrodes and eqn. (2) in which $b_{\text {eff }}$

$A_{\mathrm{B}}=b_{\mathrm{eff}} \varepsilon_{\mathrm{B}} C^{\circ}\left(D_{\mathrm{A}} / D_{\mathrm{B}}\right)^{t} t^{\frac{1}{2}}$

* To whom correspondence should be addressed. represents the effective path length of a transmission cell required to give the same absorbance as the internal reflectance cell. ${ }^{1}$ The comparable equation for external specular reflectance ${ }^{3}$ experiments would then be given by (3) where $b_{\text {eff }}$ has the same meaning as before.

$A_{\mathrm{B}}=b_{\text {eff }}(2 / \pi) \varepsilon_{\mathrm{B}} C^{\circ} D_{\mathrm{A}}^{\frac{1}{t} t^{\frac{1}{2}}}$

If the reference optically transparent electrode were $100 \%$ transparent and no light were lost during reflection from the external reflectance electrode, $b_{\text {eff }}$ in (3) could be calculated from the angle of incidence of the light beam on the electrode. The true value of $b_{\text {eff }}$ will be greater since it is possible to collect more than $80 \%$ of the incident light after a single reflection from the external reflectance electrode while the transparancy of commercially available optically transparent electrodes is about $60 \%$ for gold and $10 \%$ for platinum in the visible spectral region." Thus, from here forth the values of $b_{\text {eff }}$ discussed will be minimum values.

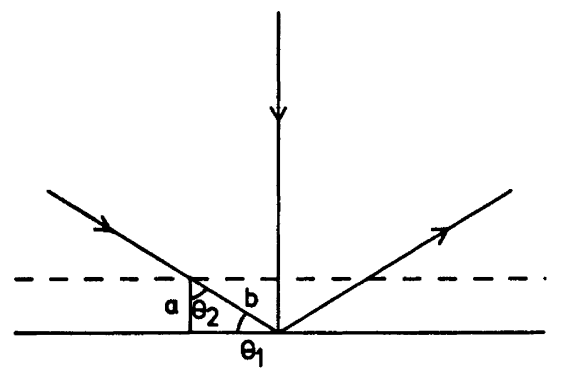

Fig. 1. Schematic diagram of light path during external reflection.

0302-4369/79/100760-03\$02.50 (C) 1979 Acta Chemica Scandinavica 

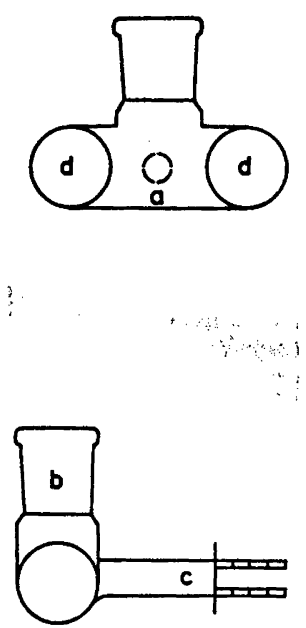

Fig. 2. Spectroelectrochemical cell, front view (a) and side view (b). Glass or quartz windows (d) and a trubore sidearm (c) for insertion of the electrode.

Referring to Fig. 1, we see that the effective pathlength is a function of the angle of incidence of light on the electrode surface. Taking the pathlength for transmission $(a)$, i.e. normal incidence, as the reference, we see that for external reflectance, the effective pathlength $\left(b_{\text {eff }}\right)$ is equal to $2 b / a$ which can be evaluated from the tangent of $\theta_{2}$. For example, $b_{\text {eff }}$ is equal to $2 \sqrt{2}$ for an angle of incidence equal to $45^{\circ}$.

The importance of $b_{\text {eff }}$ in spectroelectrochemical studies is evident from eqn. (3). When reactive intermediates are being monitored spectroelectrochemically, a 10-fold change in $b_{\text {eff }}$ would allow for the observation of an

Table 1. Absorbance and effective pathlengths for the spectroelectrochemical reduction of perylene in DMF. ${ }^{a}$

\begin{tabular}{rrrrr}
$\theta_{1}\left({ }^{\circ}\right)$ & $\tan \theta_{2}$ & $A_{\text {calc }}(\mathrm{rel})$ & $A_{\text {found }}(\mathrm{rel})^{b}$ & $b_{\text {eff }}$ \\
\hline 45 & 1.4 & 2.8 & 2.8 & 2.8 \\
75 & 4.0 & 8.0 & 6.9 & 6.9 \\
85 & 23.0 & 22 & 22 & 22 \\
\hline
\end{tabular}

a Potential steps from -1.5 to $-2.0 \mathrm{~V}$ vs. Ag/Ag ${ }^{+}$. ${ }^{b}$ Measured at $582 \mathrm{~nm} 2.0 \mathrm{~ms}$ after the potential step and referred to the value calculated for transmission based on the result at $45^{\circ}$. intermediate with a lifetime of 1/100 as great as required for the observation with the smaller value of $b_{\text {eff }}$ providing that $\varepsilon_{\mathrm{B}}$ and $D_{\mathrm{A}}$ were unchanged.

The approach taken here to increase $b_{\text {eff }}$ was to simply change the angle of incidence during specular reflectance measurements at polished platinum electrodes. Three external reflectance cells were constructed as shown in Fig. 2 with windows set at angles so that the light incident on one window would strike the planar electrode at angle $\theta_{1}$ and be reflected through the second window and onto the photomultiplier. Thus, it was possible to make measurements with $\theta_{\mathrm{a}}$ equal to approximately 45,75 and $85^{\circ}$. The reduction of perylene in $N, N$-dimethylformamide to give the stable anion radical was chosen to test the effect of $\theta$ on $b_{\text {eff }}$. Perylene anion radical shows a single absorption maximum in the visible at $582 \mathrm{~nm}$. The absorbance-time curve at $582 \mathrm{~nm}$ was recorded for $2 \mathrm{~ms}$ pulses from -1.5 to $-2.0 \mathrm{~V}$ (the peak potential for the reduction of perylene under the conditions used was found to be $-1.76 \mathrm{~V}$ vs. an $\mathrm{Ag} / \mathrm{Ag}^{+}$reference electrode.) at a frequency of $125 \mathrm{~Hz}$. The absorbance-time curves were signal averaged s (1024 curves) and the absorbance $2.0 \mathrm{~ms}$ after the potential step was determined for each of the three cells using the same electrode and identical solutions of perylene (1.0 mM) in DMF containing $\mathrm{Bu}_{4} \mathrm{NBF}_{4}$ $(0.2 \mathrm{M})$. The relative absorbances along with the values calculated from the expected effective pathlengths are summarized in Table 1.

The observed absorbance values are in very close agreement with the relative values calculated from the angle of incidence of the light on the electrode. A value of $b_{\text {eff }}$ of greater than 20 corresponds to a factor of greater than 400 in time resolution for kinetic measurements showing the great potential for external reflectance spectroelectrochemical measurements as compared to those at optically transparent electrodes. It should be pointed out that $b_{\text {eff }}$ of the order of 15 has been observed during internal reflection studies at optically transparent electrodes.1 A definite advantage of the external reflectance method is that the electrode is a normal conducting metallic surface whereas the optically transparent electrode has finite resistance which slows down the electrode response. 


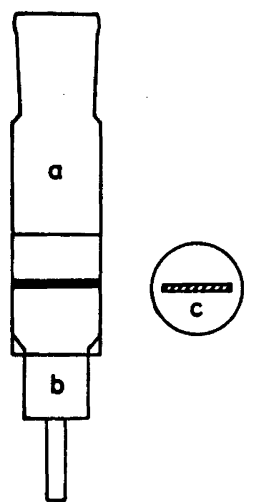

Fig. 3. Spectroelectrochemical cell consisting of a pyrex tube of inside diameter $25 \mathrm{~mm}$ (a) into which the electrode assembly (b) can be in. serted. Top view of the electrode assembly (c).

A cell of another design is illustrated in Fig. 3. In this case, the rectangular electrode is positioned in the light beam of the spectrometer from below and the angle of incidence can be adjusted to optimize the sensitivity. Although we have not made exact measurements of the angle of incidence, we estimate the optimum value to be about $1^{\circ}$. Under these conditions the calculated value of $b_{\text {eff }}$ is equal to 114 . We find this to be qualitatively the case. While absorbance time curves during $2 \mathrm{~ms}$ potential steps required signal averaging (1024 repetitions) using the larger angle cells described above, single pulse experiments of only $100 \mu \mathrm{s}$ duration gave absorbance-time curves with signal to noise ratios of the order of 8 using the perylene solutions described above. The latter indicates that $b_{\text {eff }}$ is indeed of the order of 100 for this cell and that the improvement in time resolution over the transmission case is greater than a factor of $10^{4}$.

The work reported here shows that improvements in spectroelectrochemical cell design can result in greatly enhanced sensitivity and allow measurements to be made on intermediates of lower molar extinction at shorter time steps. We do not consider the cells described here for external reflectance measurements to be optimum designs.

\section{EXPERIMENTAL}

Spectroelectrochemical cells and electrode design. The two types of cells used in this study were of particularly simple design and are illustrated in Figs. 2 and 3. The body of the cell (a) in Fig. 2 consisted of a section of $20 \mathrm{~mm}$ pyrex tubing to which a 29/43 ground glass joint (b) was attached to accomodate a stopper equipped with a counter electrode, a reference electrode and an inert gas inlet. The working electrode, after a design by Bewick, was constructed in such a way that an " $O$ " ring placed in a recess dividing the two sections could be expanded to prevent leakage by turning the back section which was attached to the front of the electrode by a screw arrangement. The electrode assembly was placed in the cell through a section of $8 \mathrm{~mm}$ trubore tubing (c) attached to the side of the cell at $90^{\circ}$ to both the cell top and to the body of the cell. The cell windows, made either of quartz or glass, were attached to the ends of the cell body at the desired angles (d). The second type cell shown in Fig. 3 consisted of a $25 \mathrm{~mm}$ (inside diameter) pyrex tube to which was attached a $29 / 43$ ground glass joint to hold the stopper containing the reference and counter electrodes along with an inert gas inlet. The electrode assembly was an enlarged version of the one described above which could be attached directly to a stand for support and adjustment of the angle of incidence.

Instrumentation. The potentiostat was a PAR 173D driven by a PAR 175 function programmer. Absorbance-time curves using the cell shown in Fig. 2 were obtained by signal averaging with a Hi-Tek Instruments signal averager $A A l$, the output from a Harrick Rapid Scan Spectrometer RSSB. Data points were measused at $5 \mu$ s intervals. Absorbance-time data points were measured at $0.5 \mu$ intervals using the cell shown in Fig. 3 with a Nicolet 1090 AR digital oscilloscope interfaced to a Hewlett Packard 9825A desk computer.

Acknowledgements. We gratefully acknowledge support for $\mathbf{E}$. A. from the Norwegian Research Council for Science and the Humanities. Special thanks are due to Dr. Ole Hammerich and Mr. P. Lund for the construction of electrodes.

\section{REFERENCES}

1. Kuwana, T. and Winograd, N. In Bard, A. J., Ed., Electroanalytical Chemistry, Dekker, New York 1974, Vol. 7.

2. Winograd, N. and Kuwana, T. Anal. Chem. 43 (1971) 252.

3. Aylmer-Kelly, A. W. B., Bewick, A., Cantrill, P. R. and Tuxford, A. M. Faraday Discuss. Chem. Soc. 56 (1973) 96.

4. Harrick Scientific Corporation, Catalogue, Ossining, New York 1977.

5. Blount, H. N., Winograd, N. and Kuwana, T. J. Phys. Chem. 74 (1970) 3231.

6. Bewick, A. Private communication.

Received June 27, 1979. 